\title{
Hypoxia and defective apoptosis drive genomic instability and tumorigenesis
}

\author{
Deirdre A. Nelson, 1,4,9 Ting-Ting Tan, ${ }^{2,4,9}$ Arnold B. Rabson, ${ }^{2,5,6,7,8}$ Diana Anderson, ${ }^{2,4}$ \\ Kurt Degenhardt, ${ }^{3,4}$ and Eileen White ${ }^{1,2,3,4,5,8,10}$ \\ ${ }^{1}$ Howard Hughes Medical Institute, ${ }^{2}$ Center for Advanced Biotechnology and Medicine, and ${ }^{3}$ Department of Molecular \\ Biology and Biochemistry, ${ }^{4}$ Rutgers University, Piscataway, New Jersey 08854, USA; ${ }^{5}$ Department of Molecular Genetics \\ and Microbiology, and ${ }^{6}$ Department of Pathology, ${ }^{7}$ University of Medicine and Dentistry of New Jersey, Robert Wood \\ Johnson Medical School, ${ }^{8}$ Cancer Institute of New Jersey, New Brunswick, New Jersey 08903, USA
}

Genomic instability is a hallmark of cancer development and progression, and characterizing the stresses that create and the mechanisms by which cells respond to genomic perturbations is essential. Here we demonstrate that antiapoptotic BCL-2 family proteins promoted tumor formation of transformed baby mouse kidney (BMK) epithelial cells by antagonizing BAX- and BAK-dependent apoptosis. Cell death in vivo correlated with hypoxia and induction of PUMA (p53 up-regulated modulator of apoptosis). Strikingly, carcinomas formed by transformed BMK cells in which apoptosis was blocked by aberrant BCL-2 family protein function displayed prevalent, highly polyploid, tumor giant cells. Examination of the transformed BMK cells in vivo revealed aberrant metaphases and ploidy changes in tumors as early as $\mathbf{9} \mathbf{d}$ after implantation, which progressed in magnitude during the tumorigenic process. An in vitro ischemia system mimicked the tumor microenvironment, and gain of BCL-2 or loss of BAX and BAK was sufficient to confer resistance to apoptosis and to allow for accumulation of polyploid cells in vitro. These data suggest that in vivo, even in cells in which p53 function is compromised, apoptosis is an essential response to hypoxia and ischemia in the tumor microenvironment and that abrogation of this response allows the survival of cells with abnormal genomes and promotes tumorigenesis.

[Keywords: Apoptosis; tumorigenesis; BCL-2; genomic instability; E1B 19K; hypoxia]

Received March 19, 2004; revised version accepted July 6, 2004.

Adenovirus and its encoded oncogenes, $E 1 A$ and $E 1 B$, possess the ability to cause normal primary rodent epithelial cells to grow into tumors (Trentin et al. 1962), and deciphering the mechanisms involved has unveiled basic processes by which human cancers develop (White 2001). The proteins encoded by the $E 1 A$ gene have a well-established role in deregulating the control of cell growth through interaction with and inhibition of the retinoblastoma protein $(\mathrm{RB})$ and its relatives, and the transcriptional coactivators p300 and CBP (CREB-binding protein). These, and perhaps other activities of E1A, stimulate cell proliferation in otherwise quiescent cells but also trigger apoptosis (Cuconati et al. 2003; Perez and White 2003). Thus, transformation of primary rodent epithelial cells in vitro is aborted in cells expressing E1A alone. Coexpression of E1B with E1A blocks apoptosis induced by E1A and enables transformation in vitro. E1B encodes two proteins, E1B 55K, which interacts with and inhibits the function of the p53 tumor suppressor pro-

\footnotetext{
${ }^{9}$ These authors contributed equally to this work.

${ }^{10}$ Corresponding author.

E-MAIL ewhite@cabm.rutgers.edu; FAX (732) 235-5795.

Article published online ahead of print. Article and publication date are at http://www.genesdev.org/cgi/doi/10.1101/gad.1204904.
}

tein, and E1B 19K, which is the adenovirus BCL-2 homolog that interacts with BAX and BAK to block apoptotic signaling through mitochondria. Both E1B proteins contribute to inhibition of apoptosis and facilitate transformation (White 2001; Cuconati and White 2002).

Inhibiting p53 function is absolutely required for transformation of primary rodent epithelial cells in vitro by E1A. First, E1A expression alone induces apoptosis and rare surviving transformants have mutated p53 (White 2001). Second, transformation in vitro by E1A is rescued by coexpression of a dominant negative mutant of p53 (Debbas and White 1993) or by using primary cells from p53-deficient mice (Lowe et al. 1994; Degenhardt et al. 2002b). Third, although coexpression of E1B 19K or BCL-2 with E1A increases transformation efficiency, they are substantially less effective than direct inactivation of p53 because E1B 19K or BCL-2 expression blocks p53-dependent apoptosis but the growth arrest function of p53 remains intact (Rao et al. 1992; Debbas and White 1993; Chiou et al. 1994). In contrast, inactivation of both BAX and BAK is not sufficient for cell transformation, as E1A alone fails to transform primary epithelial cells derived from BAX- and BAK-deficient mice, which are, however, dramatically refractory to apoptosis induction 
(Lindsten et al. 2000; Wei et al. 2001; Zong et al. 2001; Degenhardt et al. 2002b). Thus, p53 and BAX and BAK, have distinct roles in suppressing oncogenesis.

Up-regulation of antiapoptotic BCL-2 family proteins is common to many human tumors, and apoptotic defects are thought to be a universal feature of cancers (Hanahan and Weinberg 2000). Because p53 inactivation is sufficient for transformation and immortalization in vitro by E1A, whereas inactivation of mitochondrial apoptosis through the loss of both BAX and BAK is not, this suggests that blockade of the apoptotic signaling pathway through mitochondria may function independent of immortalization during tumorigenesis. In support of this, we demonstrated that E1A and mutant p53-transformed baby mouse kidney (BMK) cells that are deficient for both BAX and BAK readily form tumors in nude mice, whereas those retaining functional BAX and/or BAK do not (Degenhardt et al. 2002a). Additionally, transformed BMK cells derived from $\mathrm{p} 53$-deficient mice, which retain an intact mitochondrial apoptotic pathway, are similarly nontumorigenic (Degenhardt et al. 2002a). Finally, this study also showed that loss of apoptotic function could be selected for through loss of heterozygosity of bax or bak during tumor formation in vivo (Degenhardt et al. 2002a). These observations revealed a p53-independent apoptotic pathway that functions in epithelial cells in vivo to suppress tumorigenesis. Thus, if BAX and BAK are the functional targets of the antiapoptotic BCL-2 family proteins, then expression of BCL-2, or the adenovirus BCL-2 homolog E1B 19K, should promote tumorigenesis of E1A and mutant p53-transformed BMK cells equivalent to that of BAX and BAK deficiency. Likewise, if BAX and BAK are the only targets of BCL-2 and E1B $19 \mathrm{~K}$ necessary for promoting tumorigenesis, then their expression should not further enhance tumorigenesis of transformed BMK cells that are deficient for both BAX and BAK.

Here we report that constitutive overexpression of BCL-2 or E1B 19K promoted tumor formation in E1A and mutant p53-transformed BMK cells, but did not further enhance tumor formation in transformed BMK cells deficient for both BAX and BAK. Furthermore, transformed BMK cells were subjected to hypoxia in vivo, which was accompanied by profound apoptotic death in cells with an intact mitochondrial apoptotic pathway that was prevented by expression of BCL- 2 or by the loss of BAX and BAK. Additionally, exposure to hypoxia in vivo correlated with induction of the proapoptotic $\mathrm{BH} 3$-only protein PUMA (p53 up-regulated modulator of apoptosis), but not BIM (BCL-2-interacting mediator of cell death). Strikingly, carcinomas formed by transformed BMK cells expressing BCL-2 or E1B 19K, like those deficient for both BAX and BAK, formed prevalent polyploid tumor giant cells, whereas tumors formed from transformed BMK cells with an intact mitochondrial apoptotic pathway did not. Examination of the transformed BMK cells in vivo revealed aberrant metaphases and ploidy changes that were apparent as early as $9 \mathrm{~d}$ after injection into mice and which progressed in magnitude during the tumorigenic process. Furthermore, in vitro ischemia mim- icked the tumor microenvironment, causing induction of PUMA, but not BIM, and resulted in apoptosis in cells with an intact apoptotic pathway but not in cells with a block to the intrinsic apoptotic pathway. Additionally, ischemic treatment in vitro also promoted ploidy changes only in transformed BMK cells in which apoptosis was blocked. These data suggest that in vivo, even in cells in which p53 function is compromised, apoptosis is an essential response to environmental stresses such as hypoxia and ischemia and that abrogation of this response allows the survival of cells with abnormal genomes and promotes tumorigenesis.

\section{Results}

BCL-2 or E1B $19 \mathrm{~K}$ expression blocks apoptosis and accelerates tumorigenesis

To assess the role of antiapoptotic proteins during tumorigenesis, we used the transformed BMK cell model, where E1A and dominant negative p53 are sufficient for transformation of primary BMK epithelial cells in vitro but not for tumorigenesis in vivo (Degenhardt et al. 2002a,b). Primary BMK cells transformed with E1A and dominant negative p53 (p53DD) were derived from mice that express both BAX and BAK (W2 cells) or from mice that are deficient for both BAX and BAK (D3 cells; Degenhardt et al. 2002b). W2 cells, which express BAX and BAK and are poorly tumorigenic in nude mice (Degenhardt et al. 2002a), were then transfected with plasmids directing constitutive expression of BCL-2, the adenovirus BCL-2 homolog E1B 19K, or vector alone, selected with geneticin, and ring cloned. Multiple individual clones expressing BCL-2, E1B 19K, or vector controls were isolated. Three stable cell lines that expressed similar levels of BCL-2 or E1B 19K were then chosen for further analysis (Fig. 1A). We also generated stable cell lines expressing BCL-2, E1B 19K, and vector-only controls of BAX- and BAK-deficient BMK cells (D3 cells), as these cells are readily tumorigenic in nude mice (Degenhardt et al. 2002a). Because BCL-2 and E1B 19K should inhibit BAX- and BAK-mediated apoptosis, assessing a gain of function in the absence of BAX and BAK could reveal the existence of additional functional targets of these proteins. Again, multiple clones were isolated, and three stable cell lines that expressed similar levels of BCL-2 or E1B $19 \mathrm{~K}$ were chosen for further analysis (Fig. 1A).

To demonstrate that antiapoptotic BCL-2 family proteins functioned to block apoptosis in the stable BMK cell lines, we assayed for response to the potent apoptotic inducer staurosporine, which requires BAX or BAK to signal apoptosis through mitochondria (Degenhardt et al. 2002a). Stable cell lines expressing BCL-2, E1B 19K, or vector controls derived from transformed BMK cells that express BAX and BAK (W2), or that are deficient for both BAX and BAK (D3), were treated with staurosporine and viability was monitored by trypan blue exclusion (Fig. 1). As illustrated, vector control cells derived from W2 cells (W2.3.1 cells) were readily killed in response to stauro- 
A
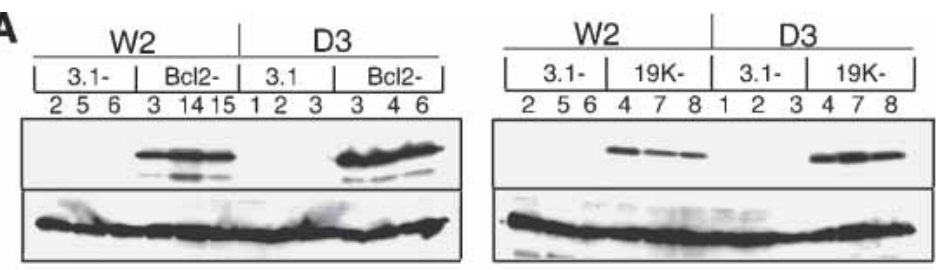

B
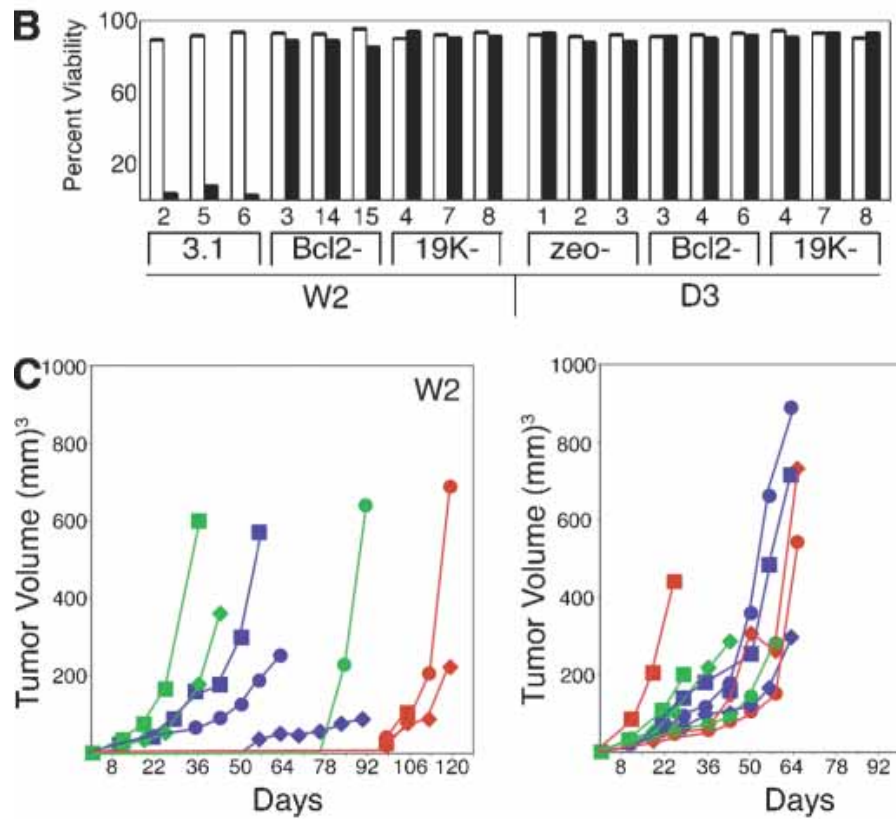

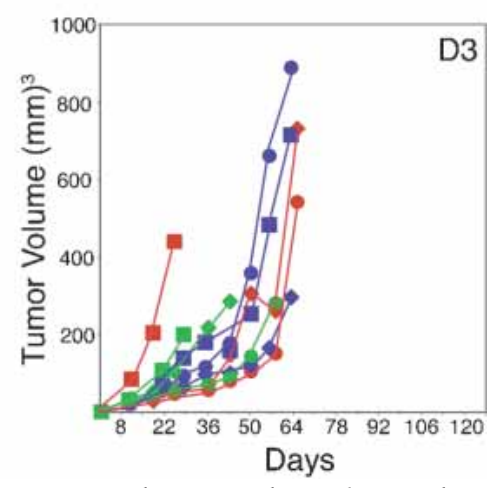

Figure 1. Antiapoptotic BCL-2 family proteins block apoptosis and promote tumor formation. $(A)$ Generation of stable cell lines. Cell extracts made from stable BMK cells that express both BAX and BAK (W2), or that are deficient for BAX and BAK (D3), were subjected to Western blotting with antibodies specific for BCL-2 (left top panel) or E1B 19K (right top panel). Note the similar expression levels of each exogenous protein in three independent clones (depicted numerically) and undetectable levels of each exogenous protein in the vector-only control cell lines (W2.3.1-2,5,6 or D3.zeo-1,2,3). Blots were then reprobed with an antibody to actin to verify nearly equivalent levels of protein in all lanes, shown below the BCL-2 and E1B $19 \mathrm{~K}$ panels. (B) BCL-2 and E1B 19K block apoptosis in response to staurosporine. Stable BMK cell lines expressing BCL-2, E1B 19K, and controls were treated with media alone (open bars) or media containing 0.4 $\mu \mathrm{M}$ staurosporine (filled bars) for $24 \mathrm{~h}$, and the viable cell number was determined by trypan blue exclusion. Results are presented as the percent of viable cells in each condition, which in each case represents the average of three independent plates. $(C) \mathrm{BCL}-2$ and $\mathrm{E} 1 \mathrm{~B}$ $19 \mathrm{~K}$ antagonize BAX and BAK to promote tumor formation. Three independent stable BMK cell lines (circles, squares, and diamonds) expressing BCL-2 (green symbols), E1B 19K (blue symbols), or controls (red symbols) were injected subcutaneously into nude mice, and tumor formation was monitored over time. Each point represents the average tumor volume for five injected animals. W2 cells, which express both BAX and BAK, are shown in the left panel. D3 cells, which are deficient for both BAX and BAK, are shown in the right panel. Note that BCL-2 or E1B 19K expression caused a profound acceleration of tumor formation in the W2 cells, whereas the kinetics of tumor formation in the D3 cells, which are deficient for both BAX and BAK, were unchanged by BCL-2 or E1B 19K expression.

sporine (Fig. 1B). This cell death, however, was almost completely blocked by the expression of BCL-2 or E1B $19 \mathrm{~K}$ (Fig. 1B). In contrast, and consistent with previous data (Wei et al. 2001; Degenhardt et al. 2002a), control transformed BMK cells derived from mice deficient for both BAX and BAK (D3.zeo cells) were resistant to cell death in response to staurosporine, and in these cells BCL-2 and E1B 19K had no additional discernable activity in this assay (Fig. 1B). Similar results were obtained using etoposide (data not shown).

W2 cells expressing BCL-2 or E1B 19K were then tested for tumorigenic potential on subcutaneous injection into nude mice. Consistent with our previous observations (Degenhardt et al. 2002a), control transformed cell lines derived from mice deficient for both BAX and BAK (D3.zeo cells) formed large tumors within 20-60 d following injection (Fig. 1C), whereas control transformed cell lines expressing BAX and BAK (W2.3.1 cells) did not form measurable tumors within that same time period, and did not do so for several months (Fig. 1C). In contrast, W2 cells expressing both BAX and BAK did readily form tumors when they also expressed either BCL-2 or E1B 19K (Fig. 1C). In these cells, however, although two of three stable cell lines expressing BCL-2 or E1B 19K rapidly formed tumors, one of each group formed tumors with somewhat delayed kinetics. Although the reason for this variable kinetics is unknown, it is not likely the result of different expression levels of exogenous antiapoptotic proteins (see Fig. 1A) and more likely reflects variability of the capacity for the function of antiapoptotic BCL-2 family proteins to be saturated or negatively regulated in cells. Further, expression of either BCL-2 or E1B 19K in transformed BMK cells that are deficient for both BAX and BAK did not alter the kinetics of tumorigenesis (Fig. 1C). These data indicate that antiapoptotic BCL-2 family proteins blocked apoptosis and accelerated tumorigenesis in nude mice in a similar fashion to the loss of proapoptotic BAX and BAK. Additionally, BCL-2 and E1B 19K did not have the ability to enhance tumor growth beyond what results from the loss of BAX and BAK function, indicating that these proteins function by inhibiting the BAX and BAK pathway during tumorigenesis.

\section{Cell death limits tumor growth in cells with an intact apoptotic pathway}

Although the vector derivatives of the transformed BMK cells that express both BAX and BAK (W2.3.1 cells) are poorly tumorigenic, tumors do eventually form from 
these cells, albeit with delayed kinetics (see Fig. 1C). To determine whether the majority of these injected cells die, or merely arrest in the mouse subcutaneum, we engineered the transformed BMK cells to express red fluorescent protein (RFP) in order to visualize the cells in vivo using a fluorescent protein whole-body imaging system. On injection into nude mice, the RFP-expressing transformed BMK cells were monitored in intact animals, and tagging each mouse allowed us to follow individual animals over time. Figure 2 shows images obtained from representative mice injected with the RFPexpressing cells. As illustrated, transformed BMK cells in which apoptosis is blocked by the gain of BCL-2 (W2.Bcl2-3), or by the loss of both BAX and BAK (D3.zeo2), showed only a slight loss of RFP signal during the first few days after injection, after which time the RFP signals expanded concomitant with tumor growth. In contrast, control transformed BMK cells with an intact apoptotic pathway (W2.3.1-5) showed progressive loss of the RFP signal for $\sim 1 \mathrm{mo}$, after which time focal RFP signals emerged and slowly expanded until overt tumors were visible in a manner suggestive of clonal expansion.

Monitoring the RFP-expressing BMK cells in intact animals suggested that control transformed BMK cells (W2.3.1-5) died after injection into nude mice unless cell death was blocked by inhibiting BAX- and BAK-mediated apoptosis. To further examine this, we excised transformed BMK cells expressing RFP from nude mice 2 and $9 \mathrm{~d}$ after subcutaneous injection, and histology of the cell masses was analyzed using hematoxylin and eosin (H\&E)-stained sections (Fig. 3, H\&E column). Consistent with the RFP data (see Fig. 2), W2.3.1-5 cells showed extensive cell death evident as a large necrotic center in the H\&E-stained sections that contained cells dying by apoptosis and necrosis on day 2 after injection (Fig. 3, inset), such that by day 9 after injection only scattered patches of BMK cells remained, indicated in the figure by arrows. In contrast, and also consistent with the RFP data, the viability of both W2.Bcl2-3 and D3.zeo-2 cells was enhanced on day 2 after injection, although these cell masses also contained necrotic regions with cells exhibiting necrotic but not apoptotic morphology (Fig. 3, inset). Furthermore, by day nine after injection, the W2.Bcl2-3 and D3.zeo-2 cell lines had both formed wellorganized tumor nodules (Fig. 3).

\section{Hypoxia limits survival in vivo}

The occurrence of large necrotic centers in the histology of the transformed BMK cell masses after injection suggested that the cells might have suffered from hypoxia on injection into mice because of the initial lack of vasculature. To test the injected cell masses for hypoxia, we performed immunohistochemistry on sections of the transformed BMK cell masses using the hypoxyprobe system, which reacts specifically with protein adducts formed in hypoxic cells and not with cells receiving adequate oxygenation from the blood supply. Additionally, we also stained adjacent sections using antibodies specific to E1A to distinguish the transformed BMK cells from host tissue. Figure 3 shows images obtained from representative animals. As illustrated, all injected transformed BMK cell lines were E1A positive and showed intense hypoxyprobe reactivity on day 2 after injection into animals, especially in cells adjacent to necrotic areas. On day 9 after injection, E1A staining again confirmed the data obtained from the H\&E sections and demonstrated that only scattered patches of W2.3.1-5 cells had survived, whereas the W2.Bcl2-3 and D3.zeo-2 cells had formed growing E1A positive tumors by this time (Fig. 3). Further, hypoxyprobe staining of sections from day 9 animals demonstrated limited reactivity in the decaying necrotic centers for the W2.3.1-5 cells and no reactivity in the emerging tumor nodules formed by the W2.Bcl2-3 and D3.zeo-2 cells, which had developed vasculature by this time (see Fig. 3). Immunohistochemistry for active caspase 3 revealed extensive caspase activation in the W2.3.1-5 cells on both day 2 and day 9, which was not evident in W2.Bcl2-3 or D3.zeo-2 cells in which apoptosis was blocked. Thus, implantation of transformed BMK cells in vivo resulted in hypoxic conditions, causing both apoptosis and necrosis. Blocking apoptosis by the gain of BCL-2 or the loss of BAX and BAK was sufficient to extend cell survival long enough to allow angiogenesis, thereby ameliorating hypoxia and promoting tumorigenesis.

\section{HIF-1 $\alpha$ and PUMA are induced in the transformed BMK cells during exposure to hypoxia in vivo}

To corroborate exposure to hypoxia in vivo, we tested the injected cells for up-regulation of the hypoxia-induc-

Figure 2. Loss of BAX and BAK or gain of BCL-2 blocks cell death in nude mice. Transformed BMK cells expressing red fluorescent protein (RFP) were injected subcutaneously into nude mice. Mice were ear-tagged and individual mice were monitored over time using a whole-body fluorescence imaging system to follow injected BMK cells. One representative animal of each cell line is shown. Note the progressive loss of RFP signal in the control transformed BMK cells (W2.3.1-5), which was largely prevented by the loss of BAX and BAK (D3.zeo-2) or the gain of BCL-2 (W2.Bcl2-3).

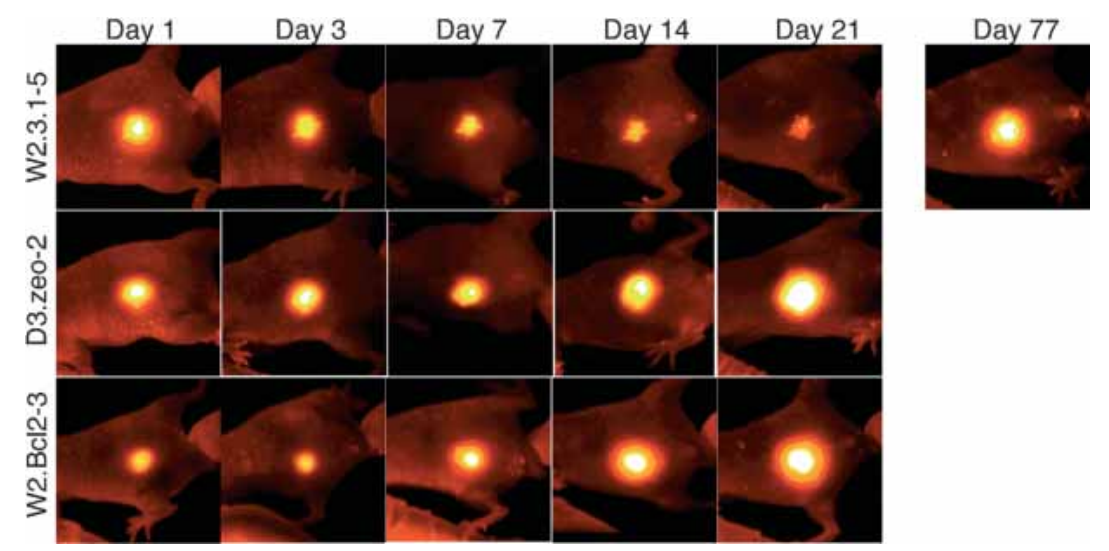




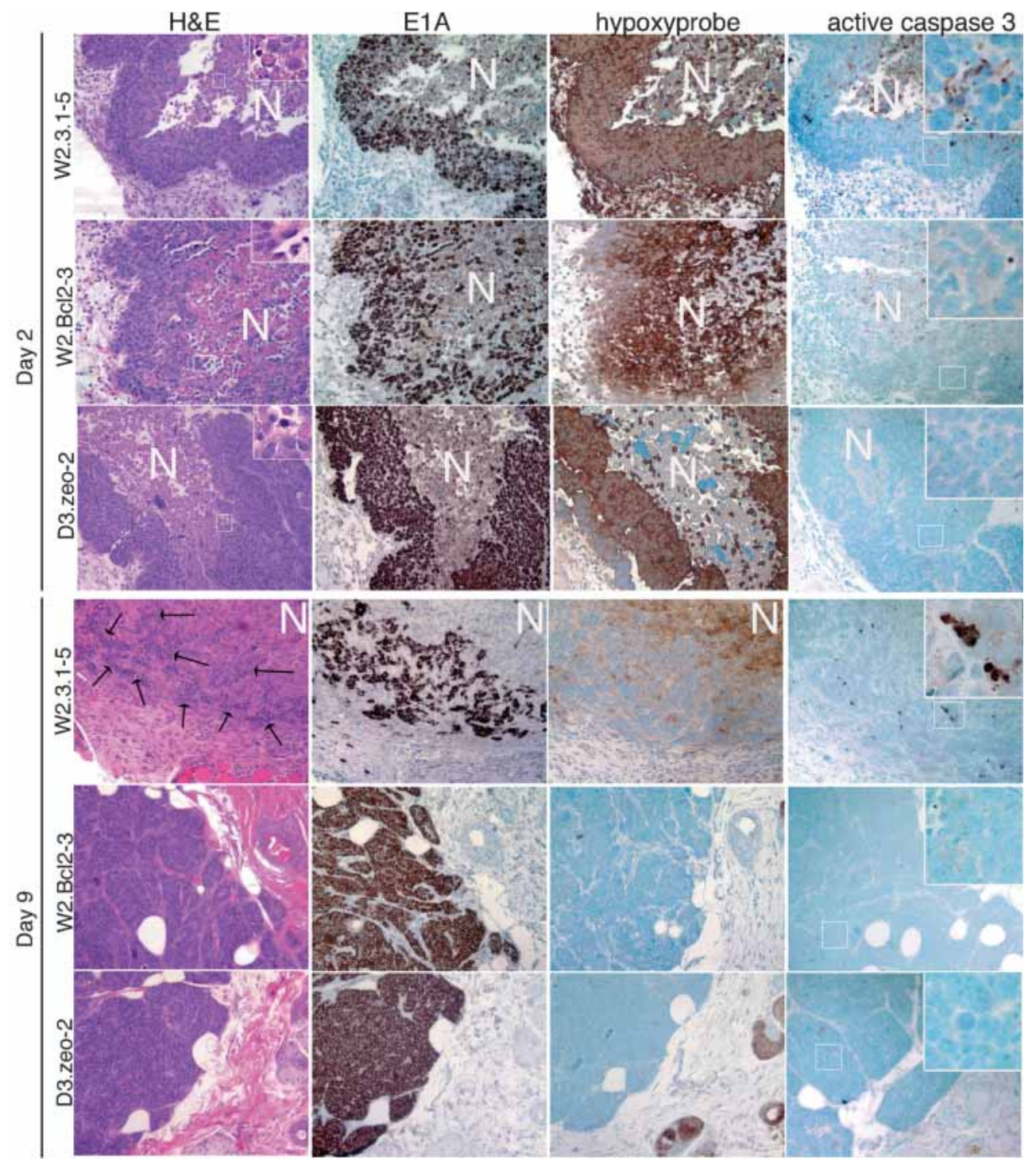

Figure 3. BMK cell death is accompanied by hypoxia in mice. Histology of the injected BMK cells reveals extensive cell death in large necrotic centers. Hematoxylin and eosin (H\&E column)-stained sections of BMK cell masses excised from animals on days 2 (top panels) and 9 (bottom panels) after injection are shown at 200×. Necrotic regions are indicated (N). Insets show W2.3.1-5 cells dying with apoptotic morphology, evident as multiple compartments of condensed chromatin in the dying cells, as well as necrotic morphology, evident as a single large patch of condensed chromatin in a dying cell. In contrast, dying W2.Bcl2-3 and D3.zeo-2 cells show only necrotic morphology (1000x). White rectangles in each panel denote areas enlarged in insets. Arrows indicate scattered patches of surviving W2.3.1-5 cells on day 9 after injection. Sections adjacent to the H\&E-stained sections were stained with antibodies specific to adenovirus E1A (E1A column) by immunohistochemistry to distinguish transformed BMK cells from host tissue (E1A reactive cells are stained brown). Sections adjacent to the E1A immunohistochemistry were developed by immunohistochemistry to hypoxyprobe adducts (hypoxyprobe column) to reveal hypoxic areas of the tumors (stained brown). Nearly adjacent sections were also developed using antibodies specific to active caspase 3 (active caspase 3 column) to reveal cells undergoing apoptosis (stained brown). Insets show active caspase 3 reactive apoptotic cells in W2.3.1-5 cell masses at 600x, and white rectangles indicate areas enlarged in the insets.

ible protein HIF-1 $\alpha$. Transformed BMK cells expressing RFP were again excised from animals 2 and 9 d after injection, and proteins extracted from these cell masses were analyzed by Western blotting using antibodies specific for HIF- $1 \alpha$ (Fig. 4A). All transformed BMK cell lines showed up-regulation of HIF- $1 \alpha$ after injection relative to extracts made from the same cell lines prior to injection. All cell lines also showed similar up-regulation of
HIF-1 $\alpha$ when subjected to hypoxia in vitro (Fig. 4A). These data confirm that the transformed BMK cell masses were subjected to hypoxia in vivo and that all BMK cell lines responded by up-regulation of HIF- $1 \alpha$ independent of their capacity for survival. Furthermore, continued induction of HIF-1 $\alpha$ in all transformed BMK cell lines on day 9, despite a loss of hypoxyprobe reactivity in the W2.Bcl2-3 and D3.zeo-2 cells, suggests that 
Nelson et al.

A

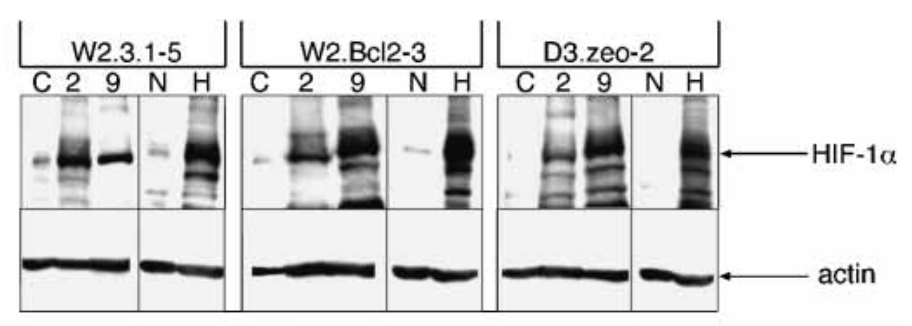

B

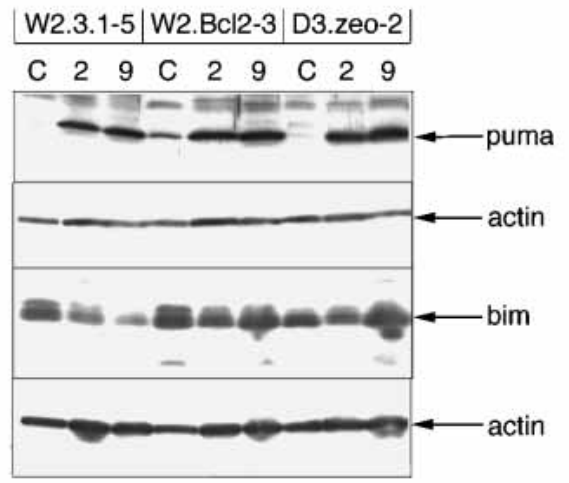

Figure 4. BMK cells subjected to hypoxia induce HIF$1 \alpha$ and PUMA in vivo. Extracts of transformed BMK cells excised from animals were subjected to Western blotting for HIF-1 $\alpha(A)$ or PUMA and BIM $(B)$. For all cell lines, $\mathrm{C}$ indicates control extracts made from the same cell lines prior to injection into animals, whereas 2 and 9 indicate extracts made from BMK cell masses excised from animals on days 2 and 9 after injection, respectively. In $A$, lanes labeled $\mathrm{N}$ and $\mathrm{H}$ indicate extracts made from cells subjected to normal oxygen culture and in vitro hypoxic culture for $12 \mathrm{~h}$, respectively. The bottom panels represent actin levels on each blot to demonstrate equivalent protein loads. (C) PUMA causes BAX- and BAK-dependent cell death. W2 cells, which contain BAX and BAK, and D2 cells, which are deficient for both BAX and BAK, were transfected with plasmids directing expression of PUMA, PUMA with a deletion of the BH3 domain (PUMA-BH3), or pCEP-4 vector control $(C)$. Twentyfour hours later, viabilities were determined as described in Materials and Methods. Note the BH3-dependent killing of the W2 cells that is completely blocked by the absence of BAX and BAK (D2 cells).

even the apparently healthy tumor nodules formed by these apoptotic resistant cell lines may still be exposed to reduced oxygen levels during the tumorigenic process. Taken together, these data suggest that hypoxic conditions in vivo are associated with apoptosis and suppression of tumor formation and that defects in this apoptotic response enhance survival long enough to allow for angiogenesis, thereby promoting tumorigenesis.

To further dissect the molecular mechanism by which hypoxic conditions in vivo may cause apoptosis, we assayed extracts made from the excised transformed BMK cells for induction of proapoptotic BH3-only proteins, some of which are known to function upstream of BAX and BAK, which might have contributed to the apoptotic cell death in vivo. Figure $4 \mathrm{~B}$ demonstrates that extracts of the transformed BMK cells excised from mice on days 2 and 9 after injection showed a dramatic induction of the $\mathrm{BH}$-only protein PUMA relative to input cells prior to injection. In contrast, the BH3-only protein BIM was not induced in the transformed BMK cells after injection into mice (Fig. 4B). Other BH3-only proteins have been

shown to function as upstream regulators of BAX and BAK or of antiapoptotic BCL-2 family proteins to regulate release of apoptogenic factors from mitochondria (Cheng et al. 2001; Zong et al. 2001; Degenhardt et al. 2002b; Letai et al. 2002). As PUMA function is known to be antagonized by BCL-2 (Nakano and Vousden 2001), we tested PUMA to determine if PUMA function is likewise dependent on BAX and BAK. Transformed BMK cells were transiently transfected with puma expression vectors and viability was monitored using cotransfection with lacZ (Fig. 4C). As illustrated, cotransfection of lacZ with puma into $\mathrm{W} 2$ cells that contain BAX and BAK caused extensive cell death relative to cotransfection with the control vector, and this cell killing was dependent on the BH3 domain of PUMA. In contrast, transformed BMK cells that are deficient for both BAX and BAK (D2 cells) were resistant to cell killing by PUMA (Fig. 4C). These data identify PUMA as a BH3-only protein induced during exposure to hypoxic conditions in vivo and suggest that PUMA may contribute to tumor suppression by causing apoptosis in response to hypoxia. 
Inhibition of apoptosis promotes tumor giant cell formation

A striking feature of the carcinomas formed by transformed BMK cells deficient for both BAX and BAK is the occurrence of many tumor giant cells in histological preparations of these tumors (Degenhardt et al. 2002a). To determine if tumor giant cell formation is a common feature of tumors with profound apoptotic defects, we examined the histology of tumors formed by the transformed BMK cells expressing BCL-2 and E1B 19K (Fig. 5A). Like the tumors formed by the BAX- and BAK-deficient transformed BMK cells, all tumors formed by W2 cells in which apoptosis was blocked by BCL-2 or E1B $19 \mathrm{~K}$ were invasive carcinomas characterized by sheets, nests, and cords of cells with high mitotic rates, extensive vascularization, areas of necrosis, and tumor giant cells (Fig. 5A; data not shown). Tumor giant cells were evident as abnormally large cells, with very large irregular nuclei that were scattered throughout the tumors and in some cases accumulated in large patches (Fig. 5A). Occasional tumor giant cells undergoing mitosis exhibited a gross excess of condensed mitotic chromosomes (Fig. 5A, insets). Immunohistochemistry for E1A expression showed extensive reactivity with the tumor giant cells, confirming their identity as aberrant transformed BMK cells (Fig. 5A). Quantitation of DNA content in tumor sections stained with YOYO-1 using confocal laser scanning microscopy and image analysis revealed that the tumor giant cells were highly polyploid with DNA contents up to over $1000 \mathrm{~N}$, which would require over eight duplications without segregation of the genome (Fig. 5A). In fact, these data are consistent with previous studies of giant cells in human tumors, where endoreplication and polytenization were shown to result

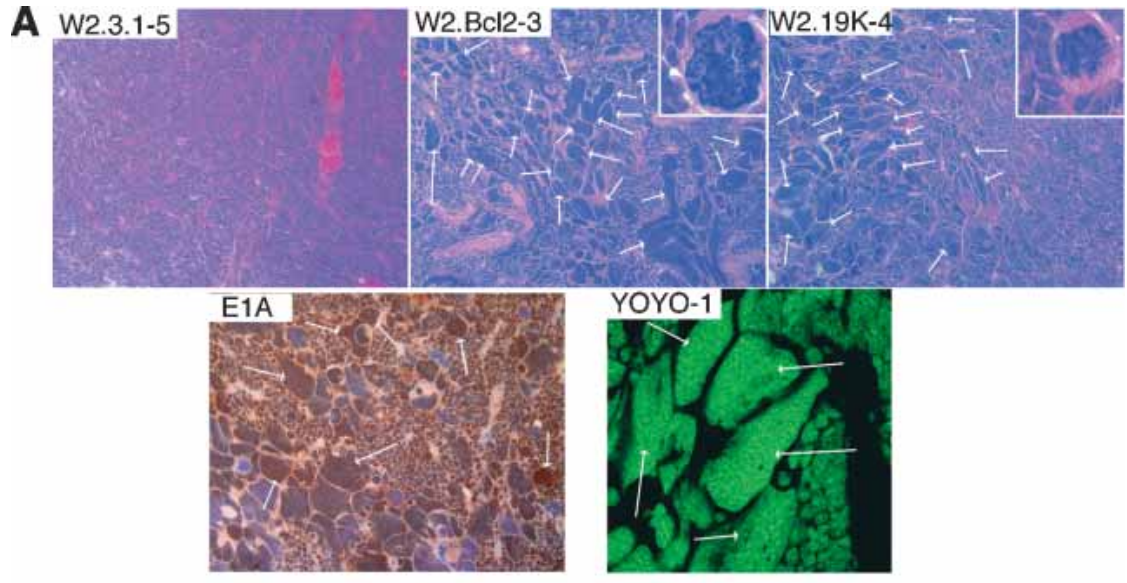

B

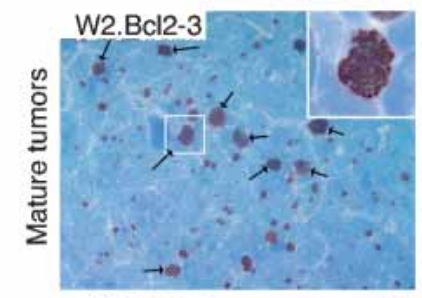

W2.3.1-5

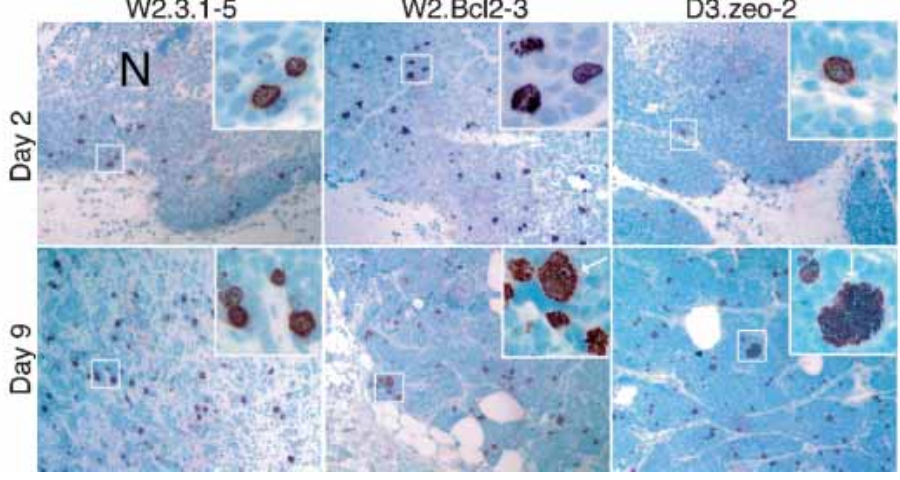

W2.Bcl2-3

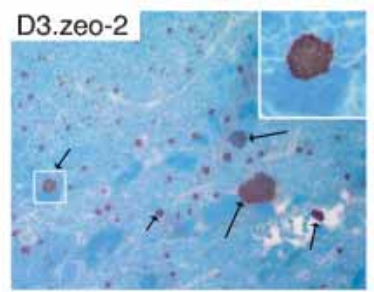

D3.zeo-2
Figure 5. Formation of tumor giant cells in tumors defective for apoptosis. $(A) \mathrm{He}$ matoxylin and eosin-stained sections reveal numerous tumor giant cells in tumors from transformed BMK cells expressing BCL-2 or E1B 19K. Typical sections of carcinomas as described in the text are shown at a magnification of $200 \times$ and highlight areas enriched for tumor giant cells in tumors from animals injected with transformed BMK cells expressing BCL-2 or E1B 19K, with insets of grossly polyploid cells in mitosis (at 1000× magnification). Several tumor giant cells in each image are indicated by white arrows. Note the absence of tumor giant cells in tumors formed by the W2.3.1-5 cells. A typical section of a tumor area enriched for tumor giant cells was also immunostained for adenovirus E1A to demonstrate that the tumor giant cells are derived from the input-transformed BMK cells. Note the numerous tumor giant cells that stain brown in the E1A immunohistochemistry, including several examples indicated by arrows. A tumor section $(20 \mu \mathrm{m})$ enriched for tumor giant cells (arrows) was stained with YOYO-1 to reveal DNA content as described in the text. This image is shown at 630x. (B) Aberrant metaphases and polyploid cells accumulate during tumor progression. Tumor sections were developed by immunohistochemistry using antibodies specific for phospho-histone $\mathrm{H} 3$ and are shown at 200x. Black arrows indicate aberrant polyploid mitotic arrays, and mitotic arrays presented at $600 \times$ in the insets are boxed. Top panels represent images of sections from mature tumors. Phosphohistone $\mathrm{H} 3$ immunohistochemistry of sections of transformed BMK cell masses excised from mice on days 2 and 9 after injection are shown in the bottom two rows

(200x). Insets in the phospho-histone H3 panels were photographed at 600x, and areas present in the insets are boxed. Grossly aberrant mitotic arrays stained for phospho-histone H3 that are evident in the W2.Bcl2-3 and D3.zeo-2, but not W2.3.1-5, cells on day 9 are indicated in the insets by white arrows. Necrotic centers are indicated $(\mathrm{N})$. 
in grossly polyploid tumor giant cells (Therman et al. 1983). In contrast, histological examination of late-forming tumors (beyond 3 mo postinjection) derived from control W2 cells expressing BAX and BAK (W2.3.1 cells) revealed no tumor giant cells (Fig. 5A). These data indicate that a block to mitochondrial apoptosis may be required for the formation of tumor giant cells and suggest that apoptosis plays a critical role in preventing this type of genomic instability.

To examine mitoses in tumors, we performed immunohistochemistry using antibodies specific for the phosphorylation of histone $\mathrm{H} 3$ on Ser 10 (Fig. 5B). As illustrated, phospho-histone $\mathrm{H} 3$ staining of the mitotic arrays in mature tumors in which apoptosis was blocked by gain of BCL-2 or loss of BAX and BAK revealed strikingly aberrant and heterogeneously sized mitotic arrays (Fig. 5B). Insets show representative large, aberrant mitotic arrays, in which cocentric rings of chromosomes were common (Fig. 5B, insets). We also examined mitoses in sections of the BMK cell masses on days 2 and 9 after injection, where we observed frequent mitoses in all transformed BMK cell lines at both times after injection, independent of their capacity for apoptosis. Furthermore, a striking majority of the mitotic cells showed metaphase chromosome arrays. Additionally, by day 9 after injection, many of the chromosome arrays in the W2.Bcl2-3 and D3.zeo-2 cell masses were disordered and heterogeneous in size, indicative of ploidy changes (Fig. $5 \mathrm{~B}$, insets). Such aberrations, however, were not evident in the W2.3.1-5 cell masses, in which the metaphase arrays were very uniform in size. Taken together, these data suggest hypoxia and/or ischemia in the tumor microenvironment interfere with normal cell cycle control and may cause mitotic checkpoint activation. Prolonged mitotic checkpoint activation is known to cause cells to exit mitosis without completing chromosome segregation or cytokinesis, in a process called mitotic slippage or adaptation (Andreassen et al. 2003). Cells with intact apoptotic pathways then die by apoptosis, whereas cells with defective apoptosis survive. Successive rounds of genome duplication followed by mitotic slippage in apoptotic-defective cells progressively alters the genomic complement, manifest as tumors with heterogeneous DNA content and including the grossly polyploid tumor giant cells. Errors in chromosome segregation in these cells would additionally allow for aberrations in chromosome number, or aneuploidy.

Defective mitochondrial apoptosis confers resistance to ischemia-induced cell death and allows

for accumulation of polyploid cells in vitro

If hypoxia- and ischemia-induced cell death are rate-limiting stimuli for transformed BMK cell tumor growth in vivo, which are overcome by gain of BCL-2 or loss of BAX and BAK, then overexpression of BCL-2, or loss of both BAX and BAK, should also protect the BMK cells from this condition in vitro. To test this, we subjected W2.3.1-5, W2.Bcl2-3, and D3.zeo-2 cells to an in vitro ischemia model system. Because isolation from the vas- culature should simultaneously deprive cells of oxygen and nutrients, these experiments were done using glucose-free media and a $1 \%$ oxygen gas mixture in the chambers. Results illustrated in Figure 6A demonstrate that the gain of BCL-2, or loss of BAX and BAK, was sufficient to maintain cell viability under these in vitro conditions, whereas the W2.3.1-5 control cells underwent progressive cell death when deprived of glucose and oxygen. Importantly, consistent with our in vivo data (see Fig. 4B) PUMA, but not BIM, was induced during the ischemic treatment in vitro (Fig. 6B). These data indicate that abrogation of apoptosis by BCL-2 family proteins is sufficient to protect cells from ischemia-induced apoptosis and suggest that PUMA induction contributes to this ischemic cell death.

In nude mice, only tumors formed from transformed BMK cells in which BAX- and BAK-mediated apoptosis was blocked developed tumor giant cells (see Fig. 5), suggesting that a block to the intrinsic apoptotic pathway may be required to manifest this type of genomic instability in vivo. Hence, we determined if gain of BCL-2, or loss of BAX and BAK, was sufficient to promote polyploidy in response to ischemia in vitro. Transformed BMK cells were subjected to the ischemia model system for $2 \mathrm{~d}$ and then transferred to normal culture conditions where they were allowed to recover for an additional $3 \mathrm{~d}$. Flow cytometry shows that the ischemic treatment caused a decrease in the population of diploid cells and a concomitant increase in cells having tetraploid and even polyploid DNA contents (Fig. 6C). All cell lines in ischemia also displayed an increase in the G2/M population relative to the G0/G1 population, indicative of mitotic checkpoint activation (Fig. 6B, middle panels). Control transformed BMK cells (W2.3.1-5) also underwent extensive apoptosis, evident in the figure as accumulation of a large sub- $2 \mathrm{~N}$ peak, and on recovery these cells did not accumulate polyploids. In striking contrast, however, transformed BMK cells in which apoptosis was blocked by the gain of BCL-2 (W2.Bcl2-3), or by the loss of BAX and BAK (D3.zeo-2), survived the induction of polyploidy and accumulated a population of cells with greater than $4 \mathrm{~N}$ DNA content. Insets show representative images of 4'6-diamino-2-phenylindole dihydrochloride (DAPI)-stained nuclei from the transformed BMK cells. As illustrated, accumulation of multinucleated or giant nuclei containing cells was restricted to the W2.Bcl2-3 and D3.zeo-2 cells, in which apoptosis was blocked by the gain of BCL-2 or the loss of both BAX and BAK, respectively. Additional experiments revealed that although hypoxia alone or glucose deprivation alone could cause some ploidy alterations, the combination of hypoxia and glucose deprivation in the ischemic model system was much more potent at driving ploidy changes in this system (data not shown). To further investigate the ploidy status of the multi- and giant-nucleated cells that accumulated in the W2.Bcl2-3 and D3.zeo-2 cells, we also analyzed DNA content in these cells and in untreated control cells by confocal laser scanning microscopy (Fig. 6D). Images of representative giant-nucleated cells are shown, and quantitation of the total DNA con- 
A

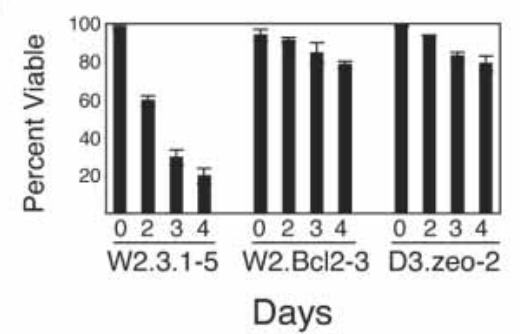

B
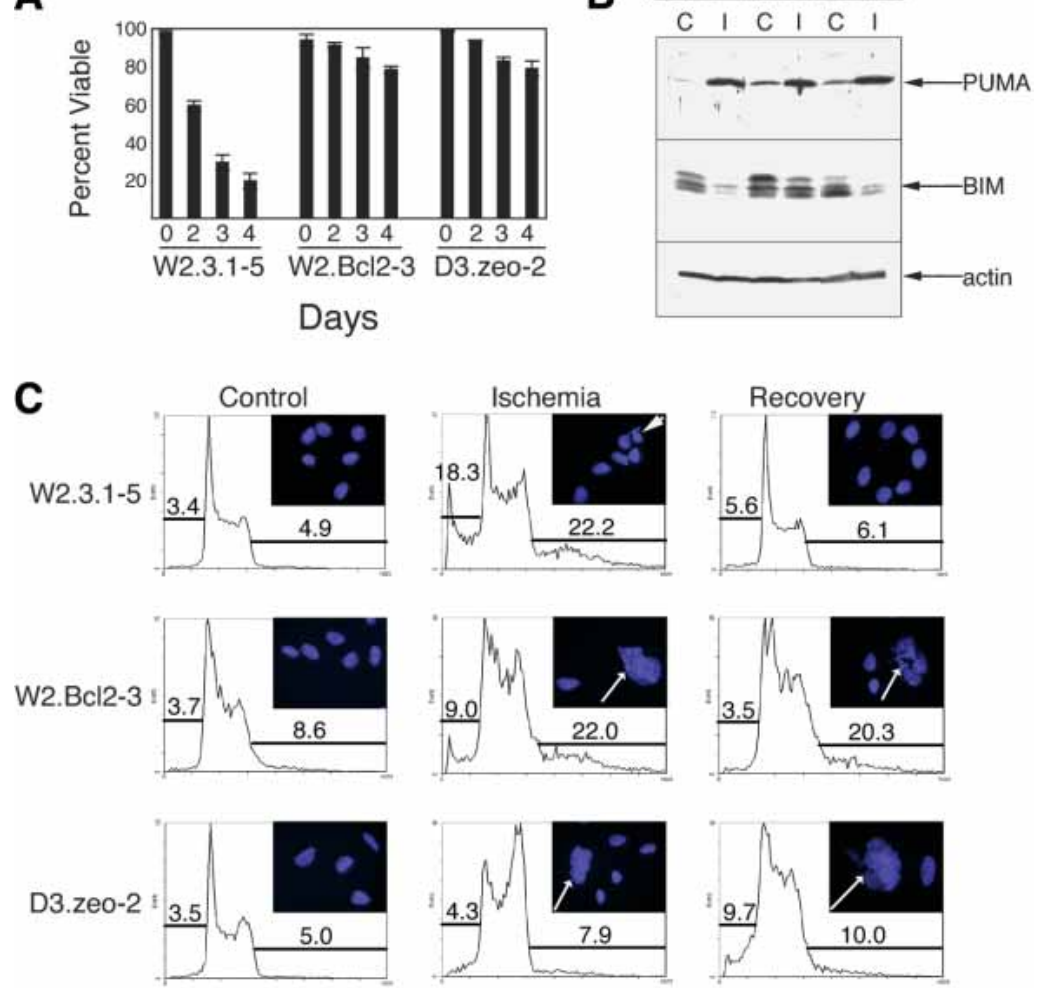

D
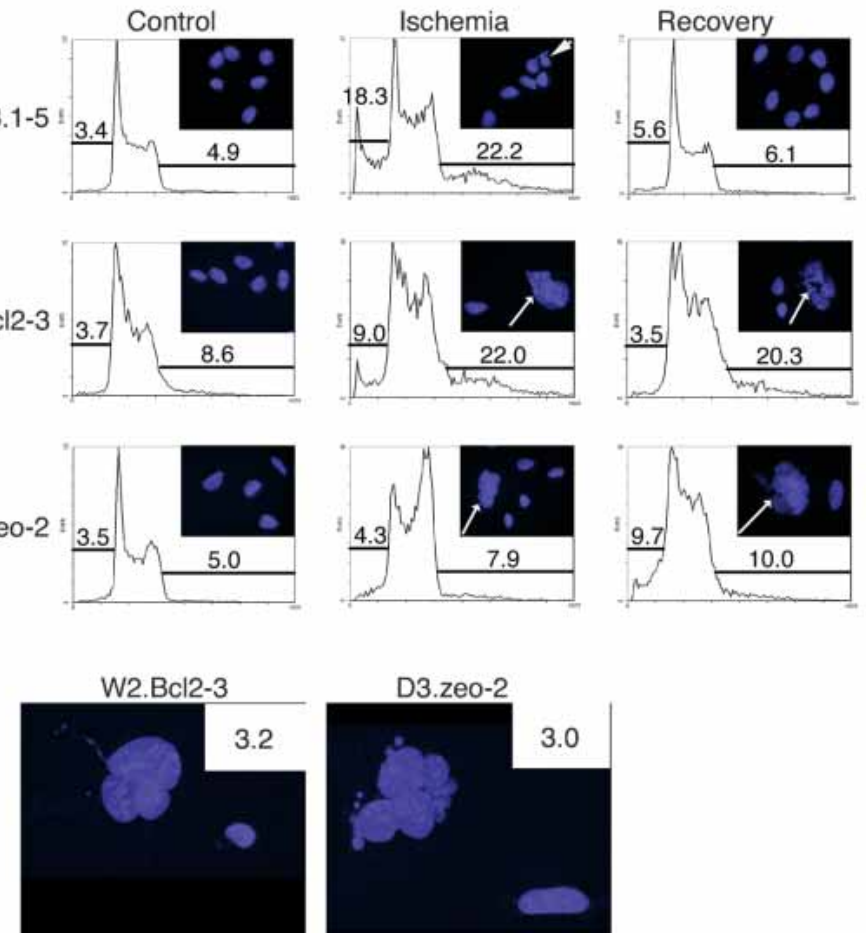

Figure 6. Defective apoptosis confers resistance to ischemia-induced cell death and allows for the accumulation of polyploid cells in response to ischemia in vitro. (A) Gain of BCL-2 or loss of BAX and BAK blocks ischemia-induced cell death. Transformed BMK cell lines were subjected to in vitro ischemic culture conditions and viabilities were determined on the indicated days. Results of triplicates are plotted for each time point. $(B)$ PUMA is induced by ischemia in vitro. Cell extracts made from cells cultured for $24 \mathrm{~h}$ in normal control conditions (C), or in ischemic culture conditions (I), were immunoblotted for PUMA, BIM, and actin. Note the induction of PUMA, but not $\mathrm{BIM}$, in ischemic culture conditions. $(C, D)$ Gain of BCL-2 or loss of BAX and BAK allows for accumulation of polyploid cells in response to ischemia. Transformed BMK cell lines were subjected to ischemic culture conditions for $2 \mathrm{~d}$ and then returned to normal culture conditions in complete media for $3 \mathrm{~d}$. (C) Cells were collected prior to ischemia, following $2 \mathrm{~d}$ of ischemia, and after ischemia with an additional $3 \mathrm{~d}$ of recovery on return to normal culture conditions and analyzed by flow cytometry. Insets illustrate typical nuclear morphology in each condition visualized by DAPI staining of cells on coverslips. Arrows indicate typical multi-/giant-nuclei in W2.Bcl2-3 and D3.zeo-2 cells, and the arrowhead indicates a W2.3.1-5 cell dying in hypoxia. (D) Representative polyploid cells stained with DAPI were also imaged using confocal laser scanning microscopy. In each panel, a nucleus of normal size cell is included in the picture for comparison. Ratios of the total DNA content of the polyploid cells to the total DNA content of the control cells are included in the insets for each panel.

tent of the multinucleate versus control cells confirmed that these cells are polyploid, indicated in the figure as a ratio of the total DNA content. Note that for both W2.Bcl2-3 and D3.zeo-2 the total DNA content of the multi- or giant-nucleated cells was approximately three times that of the control cells, indicative of an approximate $6 \mathrm{~N}$ DNA content and consistent with the FACS analysis (Fig. 6C). Taken together, these data indicate that ischemia interferes with normal cell cycle control, resulting in mitotic checkpoint activation. Metaphase arrest, however, is transient, resulting in mitotic slippage, or adaptation. In W2 cells, this triggers apoptosis through BAX and BAK, thereby eliminating polyploid cells. In W2 cells expressing BCL- 2 and in D 3 cells where BAX and BAK-dependent apoptosis is blocked, however, polyploid cells can survive and accumulate in response to ischemia, thereby contributing to the genomic instability that characterizes cancer progression.

\section{Discussion}

The role of deregulation of apoptosis in oncogenesis is complex and linked to successive and interdependent ge- netic events that gradually lead to tumor formation. Some genetic events, such as inactivation of the RB pathway, that are probably required for the development of most human cancers, also activate apoptosis (White 1994; Hanahan and Weinberg 2000). Another common genetic event in human cancer is the inactivation of the p53 pathway, which causes loss of checkpoint regulation to induce cell cycle arrest or apoptosis in response to DNA damage, hypoxia, oncogene activation, and a myriad of other cellular stresses (Levine 1997). Still other oncogenic events, exemplified by BCL-2 translocation and overexpression, directly block apoptotic signaling through mitochondria (Cory and Adams 2002). Presumably this functional interplay between activation and suppression of apoptosis during oncogenesis derives from interdependent events selected for during tumorigenesis in a process facilitated by genetic instability.

Despite these seminal observations, the relationship between inactivation of the RB and p53 pathways and inhibition of apoptosis in oncogenesis has not always been entirely clear. We now know that suppressing signaling of apoptosis through BAX and BAK appears to be dispensable for transformation by E1A and mutant p53 
in vitro but is required for efficient tumor growth in vivo (Degenhardt et al. 2002a). We show here that both BCL-2 and the adenovirus BCL-2 homolog E1B 19K also functioned to promote tumorigenesis in vivo. Additionally, we found no evidence for a gain of function for either BCL-2 or E1B 19K during tumor formation in the absence of BAX and BAK. Both BCL-2 and E1B 19K have been reported to interact with other proteins besides $\mathrm{BAX}$ and BAK, including proapoptotic BH3-only BCL-2 family members (Puthalakath and Strasser 2002) that function upstream of and require BAX or BAK for their apoptotic function (Cheng et al. 2001; Zong et al. 2001), as well as other proteins that are not members of the BCL-2 family (Reed 1998). However, because expression of either BCL-2 or E1B 19K in BAX- and BAK-deficient cells did not accelerate tumorigenesis over that of BAX and BAK deficiency alone, all of the tumorigenic activity of these proteins can be explained by inhibition of apoptosis signaled through BAX and BAK. This could be accomplished by direct inhibition of BAX and BAK, as is the case for E1B 19K (Cuconati et al. 2002), or by preventing BAX and BAK activation indirectly through inhibition of $\mathrm{BH} 3$-only proteins, which is likely the case for BCL-2 (Cheng et al. 2001).

Upon subcutaneous injection into nude mice, transformed BMK cells were subjected to hypoxia, and likely to ischemia as well. In this tumor microenvironment, many cells died with apparent necrotic morphology independent of their apoptotic propensity. W2 cell lines with an intact apoptotic ability also exhibited extensive apoptosis following injection that continued to eliminate nearly all of the injected cells over several weeks. Cell lines in which apoptosis was blocked by the gain of BCL-2 or the loss of both BAX and BAK, however, quickly recovered from hypoxia or ischemia and progressed to form tumors. Consistent with these data, cMyc sensitization to oxygen-deprivation-induced cell death was also recently shown to require BAX and BAK, but not p53 (Brunelle et al. 2004). The proapoptotic BH3only protein PUMA was induced in all implanted cell lines on days 2 and 9 after injection and was also induced in an ischemic culture model system in vitro. As PUMAmediated apoptosis is antagonized by BCL-2 (Nakano and Vousden 2001), and requires BAX and BAK (Fig. 4), this could account for apoptosis only in the W2 cells and not in the BCL-2-expressing or BAX-and BAK-deficient cells. PUMA is a potent cell killer induced by both p53dependent and p53-independent apoptotic stimuli (Han et al. 2001; Nakano and Vousden 2001; Yu et al. 2001; Jeffers et al. 2003; Reimertz et al. 2003; Villunger et al. 2003). In previous experiments, we demonstrated that BAX- and BAK-dependent tumor suppression of transformed BMK cells in nude mice is p53 independent, as BMK cells transformed with E1A and p53DD or BMK cells from $\mathrm{p} 53^{-/-}$mice transformed with E1A alone were similarly nontumorigenic in nude mice (Degenhardt et al. 2002a). Ischemia imparts endoplasmic reticulum (ER) stress on cells, and ER stress has recently been shown to cause p53-independent induction of PUMA (Reimertz et al. 2003), suggesting factor deprivation and ER stress as potential mechanisms by which an ischemic microenvironment could cause p53-independent PUMA induction. Thus, in vivo, even in cells where p53 function is compromised, PUMA-induced apoptosis would function to eliminate displaced cells without proper access to the blood supply, a condition common in solid tumors. This apoptotic response could synergize with other apoptotic effectors in vivo, which would likely include other BH3only proteins activated in response to both p53-dependent and p53-independent stresses, depending on the p53 status of the cells. Abrogation of the apoptotic response to hypoxia or ischemia, however, as through the gain of antiapoptotic BCL-2 family proteins or through loss of proapoptotic BAX and BAK, would provide a mechanism by which aberrant cell survival would contribute to tumor formation (Fig. 7).

The occurrence of tumor giant cells was a striking phenotype of the tumors formed by transformed BMK cells with a mitochondrial apoptotic block, either by loss of BAX and BAK (Degenhardt et al. 2002a) or through overexpression of BCL-2 or E1B 19K (see Fig. 5). Tumor giant cells in human tumors are associated with polyploidy and aneuploidy, and the tumor giant cells observed here were clearly polyploid. Polyploidy results from mitotic checkpoint failure or mitotic slippage and endoreplication of cellular chromosomal DNA and has been purported to be a prerequisite to the genomic instability and aneuploidy associated with the genesis and progression of human tumors (Cleveland et al. 2003; Storchova and Pellman 2004). In fact, naturally occurring polyploidy, especially in plants, is a common mechanism for the generation of genetic diversity and may have contributed to early evolution of the vertebrate lineage (Storchova and Pellman 2004). In these cases, divergence results from chromosome rearrangement that is facilitated by polyploidy. In mammals, however, most cells are diploid, and have elaborate checkpoints to prevent tetraploidy and polyploidy. Thus, cancers require abrogation

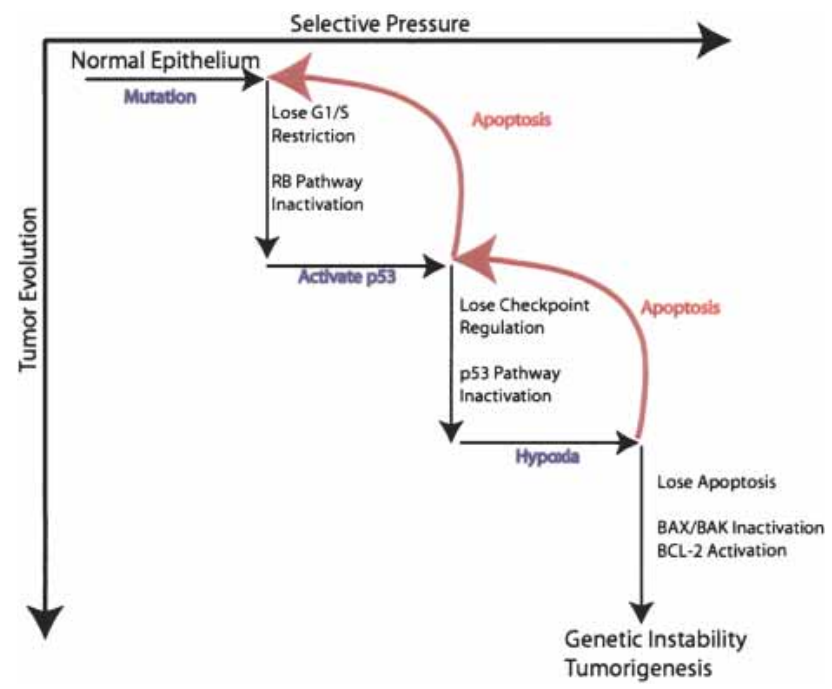

Figure 7. Model for tumor selection and progression. See text for explanation. 
of these checkpoints for survival and progression. Once achieved, however, polyploidy can contribute to tumor progression in a variety of ways, including generation of chromosomal aberrations resulting in gene duplication or loss and altered epigenetic chromatin changes that facilitate gene silencing, inevitably allowing for evasion of normal tumor suppression and increased rates of adaptation (Cleveland et al. 2003; Storchova and Pellman 2004).

In this report, we show that ischemia or hypoxia promoted polyploidy, both in vitro and in vivo. Further, only cells with defects in the intrinsic apoptotic pathway survived these ploidy changes, similar to the requirement for an apoptotic block to overcome the mitotic spindle checkpoint response to aberrant ploidy in p53deficient cell lines exposed to mitotic spindle inhibitors (Minn et al. 1996). In situ, transformed cells would encounter many obstacles to tumor growth and progression, including hypoxia and nutrient deprivation, but also including changes in cell-cell and cell-matrix interactions, inflammatory and growth inhibitory cytokines, and cell cycle checkpoint controls, to name a few. Thus, acquisition of mutations that suppress apoptosis is considered a hallmark of cancer (Hanahan and Weinberg 2000). Indeed, hypoxia in tumors is associated with poor prognosis and may promote tumor progression (Graeber et al. 1996; Harris 2002), although the basis for this is not entirely clear. Our data indicate that by promoting polyploidy, one way in which hypoxia, or ischemia, may contribute to tumor progression is by directly promoting genetic instability, thereby accelerating the incidence of genetic changes, which would then be selected for by the tumor microenvironment. Surviving these ploidy changes would, however, require selection for loss of the normal apoptotic response. In fact, human tumors are often aneuploid, with near triploid DNA contents common, and near tetraploid DNA contents also occurring (Storchova and Pellman 2004), and aneuploidy correlates with poor prognosis in many human tumors (Southern et al. 1996; Baba et al. 2002; Bocking and Nguyen 2004; Sudbo et al. 2004). Additionally, BCL-2 expression is correlated with aberrant nuclear morphology and aneuploidy in many human cancers, including leukemia (Eksioglu-Demiralp et al. 1999) and prostate cancer (Maffini et al. 2001), and often correlates with therapeutic unresponsiveness. Thus, one important role for apoptosis in tumor suppression would be to eliminate genetically unstable cells by a p53-independent mechanism, and abrogation of this response by aberrant Bcl-2 family function would simultaneously promote tumor formation and provide a mechanism for evasion of effective therapeutics.

\section{Materials and methods}

Generation of stable cell lines

Derivation of BMK cell lines was described (Degenhardt et al. 2002b). Stable BMK cells expressing human BCL-2, E1B 19K, or vector-only controls were derived by electroporation with pCDNA3.1-hBcl-2, pCDNA3.1/V5/His-TOPO19K (Kasof et al. 1998), or selection vector only (W2, pCDNA3.1; D3, pCDNA3.1zeo, InVitrogen), respectively, followed by selection (W2, geneticin; D3, zeocin) and ring cloning. All stable D3 cells were transfected with pCDNA3.1zeo and selected with zeocin. Multiple clones from each condition were expanded and characterized. For RFP expression, cells were cotransfected with the pDsRed2-C1 (Clontech) and pCDNA3.1zeo (W2.3.1-5, InVitrogen) or pPUR (D3.zeo-2, BD Biosciences) vectors, followed by selection with zeocin or puromycin, respectively, and ring cloning. For each cell line, three individual clones were characterized in animals for fluorescence and tumor growth, and one individual clone was selected for further analysis. All cell lines were maintained in DMEM (Gibco/InVitrogen) containing 10\% fetal bovine serum (FBS).

\section{Treatment of cells in culture}

For drug treatment, cells were plated and allowed to adhere overnight. Media was then aspirated and replaced with media containing vehicle alone or media containing staurosporine (Sigma-Aldrich). As a model for in vitro ischemia, cells were plated and allowed to adhere overnight, and media was replaced with glucose-free DMEM (Gibco/InVitrogen) containing 10\% FBS. Cells in standard tissue culture dishes were then placed in modular incubator chambers (Billups-Rothenberg), and air inside the chambers was replaced with a defined gas mixture containing $1 \%$ oxygen, $5 \% \mathrm{CO}_{2}$, and $94 \% \mathrm{~N}_{2}$ (AirGas). For viability studies, after treatment, cells were collected by centrifugation of the pooled culture supernatants and adherent cells, which were removed by standard trypsin-EDTA treatments. Cells were resuspended in phosphate buffered saline (PBS), diluted 1:1 in $0.25 \%$ trypan blue (Gibco/Invitrogen), and counted with a hemacytometer. For Western blotting, collected cells were lysed and analyzed as described following. For transient transfections, $9 \mu \mathrm{g}$ of various plasmid DNAs were cotransfected into cells with $3 \mu \mathrm{g}$ of pCMV- $\beta$-gal as a reporter plasmid and viability was monitored by expression of the reporter gene as previously described (Han et al. 1996). Plasmids used were pHA-PUMA and pHA-PUMA- $\Delta$-BH3 (Yu et al. 2001), which were generously provided by Dr. Bert Vogelstein, and pCEP4 as the parental control vector.

\section{Tumor formation in nude mice}

Tumor formation in nude mice by subcutaneous injection using $10^{7}$ cells in $0.1 \mathrm{~mL}$ PBS was performed essentially as described previously (Degenhardt et al. 2002a). Briefly, $10^{7}$ cells were injected in each of five mice for each cell line and tumors were measured regularly. Tumor volumes were calculated using the formula length (millimeters) $\times$ width $^{2}(\text { millimeters })^{2} / 2$. For graphing, each point represents the mean value for all five mice in each group. Some samples for histology were treated as described (Degenhardt et al. 2002a) by the Mutant Mouse HistoPathology Laboratory (University of California, Davis) and some samples were fixed with buffered formalin (Fisher Scientific) and processed by the Tissue Analytical Services (Cancer Institute of New Jersey). To visualize RFP-expressing cells in mice, we imaged animals using the Illumitool imaging and camera system (Lightools Research).

\section{Antibodies and Western blotting}

Cell or tissue extracts made in Laemmli buffer (for BCL-2, E1B $19 \mathrm{~K}$, and HIF- $1 \alpha$ ) or in Triton X-100 buffer containing $50 \mathrm{mM}$ 
Tris-Cl (pH 7.4), 100 mM NaCl, 0.1 mM EGTA, 0.1 mM EDTA, $1 \%$ Triton $\mathrm{X}-100,1 \mathrm{mM}$ sodium orthovanadate, $20 \mathrm{mM}$ sodium fluoride, $30 \mathrm{mM} \beta$-glycerophosphate, $1 \mathrm{mM}$ phenylmethylsulfonyl fluoride, and protease inhibitor cocktail complete (Roche; for PUMA and BIM) were separated by SDS-PAGE (30 $\mu \mathrm{g}$ protein) and transferred to PVDF (polyvinylidene fluoride) membranes (Schleicher and Schuell Inc.). Blots were incubated with primary antibodies, and developed with horseradish peroxidaseconjugated secondary antibodies using the ECL system (Amersham BioSciences). Primary antibodies used were hamster antihuman BCL-2 (BD-Pharmingen); rabbit polyclonal anti-E1B 19K (Sundararajan and White 2001), mouse anti-actin (Oncogene Research Products), rabbit anti-mouse HIF- $1 \alpha$ (Cayman), rabbit anti-BIM (Axxora), or rabbit polyclonal antisera raised in our lab against a GST-human PUMA fusion protein encoding a 102amino acid region common to PUMA- $\alpha$ and PUMA- $\beta$.

\section{Immunohistochemistry}

Immunohistochemistry was performed on formalin-fixed paraffin sections using pooled monoclonal antibodies (M58 + M73) to adenovirus E1A (Abcam), rabbit anti-active caspase 3 (9661, Cell Signaling Technologies), or rabbit anti-phospho-histone H3 (Upstate Biotechnology) and were developed with the DAKO LSAB2 System, Peroxidase (DAKO) using the 3,3' diaminobenzidine (DAB) chromophore that forms a brown precipitate at the site of immunolocalization according to the manufacturer's recommendations. Detection of hypoxia was done using the hypoxyprobe-1 kit (Chemicon International) for detection of hypoxic cells in animals as per the manufacturer's recommendations. Briefly, mice were injected intraperitoneally with $60 \mathrm{mg} /$ $\mathrm{kg}$ hypoxyprobe (pimonidazole) diluted in PBS and sacrificed 45 min later. Tumors were excised, fixed with buffered formalin (Fisher Scientific), and embedded in paraffin, and serial sections were obtained. Sections were developed as per the manufacturer's recommendations for immunohistochemical detection of the pimonidazole-proteins adducts, which form specifically in hypoxic cells and not in well-oxygenated tissue. Tissue sections for both detection systems were counterstained with Aqua Hematoxylin (Innovex BioScience) prior to mounting.

\section{Flow cytometry}

Determination of DNA content by FACS analysis of $10^{4}$ cells stained with propidium iodide per condition was performed by the Analytical Cytometry Unit (Cancer Institute of New Jersey) using a Cytomics FC 500 (Beckman Coulter).

\section{Confocal laser scanning microscopy}

Confocal laser scanning microscopy was done by the Neuroscience Imaging Facility (W.M. Keck Center for Collaborative Neuroscience). Cultured cells were fixed with $2 \%$ paraformaldehyde and stained with DAPI. Sections of paraffin-embedded tissue were processed and stained with YOYO-1 (Molecular Probes) as described (Liu et al. 1997). Quantitation of total DNA content in DAPI-stained cultured cells and YOYO-1-stained tumor cells in sections was done using the IMARIS program.

\section{Acknowledgments}

We thank Dr. Celine Gelinas for the generous gift of the BCL-2 expression plasmid, Dr. Bert Vogelstein and Dr. Karen Vousden for puma expression constructs, Julie Friedman for histology, and Dr. Noriko Kane-Goldsmith for assistance with the confo- cal microscopy and analysis. We also thank Thomasina Sharkey for assistance with preparation of the manuscript. This work was supported by the Howard Hughes Medical Institute and a grant from the National Institutes of Health (R37CA53370) to Dr. Eileen White.

The publication costs of this article were defrayed in part by payment of page charges. This article must therefore be hereby marked "advertisement" in accordance with 18 USC section 1734 solely to indicate this fact.

\section{References}

Andreassen, P.R., Lohez, O.D., and Margolis, R.L. 2003. G2 and spindle assembly checkpoint adaptation, and tetraploidy arrest: Implications for intrinsic and chemically induced genomic instability. Mutat. Res. 532: 245-253.

Baba, H., Korenaga, D., Kakeji, Y., Haraguchi, M., Okamura, T., and Maehara, Y. 2002. DNA ploidy and its clinical implications in gastric cancer. Surgery 131: S63-S70.

Bocking, A. and Nguyen, V.Q. 2004. Diagnostic and prognostic use of DNA image cytometry in cervical squamous intraepithelial lesions and invasive carcinoma. Cancer 102: 41-54.

Brunelle, J.K., Santore, M.T., Budinger, G.R.S., Tang, Y., Barrett, T.A., Zong, W.X., Kandel, E., Keith, B., Simon, M.C., Thompson, C.B., et al. 2004. c-Myc sensitization to oxygen deprivation-induced cell death is dependent on Bax/Bak, but is independent of p53 and hypoxia-inducible factor-1. J. Biol. Chem. 279: 4305-4312.

Cheng, E., Wei, M., Weiler, S., Flavell, R., Mak, T., Lindsten, T., and Korsmeyer, S. 2001. BCL-2, BCL- $\mathrm{X}_{\mathrm{L}}$ sequester BH3 domain-only molecules preventing BAX- and BAK-mediated mitochondrial apoptosis. Mol. Cell 8: 705-711.

Chiou, S.-K., Rao, L., and White, E. 1994. Bcl-2 blocks p53dependent apoptosis. Mol. Cell. Biol. 14: 2556-2563.

Cleveland, D.W., Mao, Y., and Sullivan, K.F. 2003. Centromeres and kinetochores: From epigenetics to mitotic checkpoint signaling. Cell 112: 407-421.

Cory, S. and Adams, J.M. 2002. The Bcl2 family: Regulators of the cellular life-or-death switch. Nat. Rev. Cancer 2: 647656.

Cuconati, A. and White, E. 2002. Viral homologues of Bcl-2: Role of apoptosis in the regulation of virus infection. Genes \& Dev. 16: 2465-2478.

Cuconati, A., Degenhardt, K., Sundararajan, R., Anschel, A., and White, E. 2002. BAK and BAX function to limit adenovirus replication through apoptosis induction. J. Virol. 76: 45474558.

Cuconati, A., Mukherjee, C., Perez, D., and White, E. 2003. DNA damage response and MCL-1 destruction initiate apoptosis in adenovirus-infected cells. Genes \& Dev. 23: 2922 2932.

Debbas, M. and White, E. 1993. Wild-type p53 mediates apoptosis by E1A which is inhibited by E1B. Genes \& Dev. 7: 546554.

Degenhardt, K., Chen, G., Lindsten, T., and White, E. 2002a. Bax and Bak mediate p53-independent suppression of tumorigenesis. Cancer Cell 2: 193-203.

Degenhardt, K., Sundararajan, R., Lindsten, T., Thompson, C.B., and White, E. 2002b. Bax and Bak independently promote cytochrome-c release from mitochondria. J. Biol. Chem. 277: 14127-14134.

Eksioglu-Demiralp, E., Budak-Alpdogan, T., Alpdogan, O., Atalay, A., Ratip, S., Oz, D., Bayik, M., and Akoglu, T. 1999. Double G0/G1 peak in the DNA histogram of aberrant marker positive acute leukemia patients is associated with a 
poor clinical outcome. Leuk. Lymphoma 33: 567-572.

Graeber, T.G., Osmanian, C., Jacks, T., Housman, D.E., Koch, C.J., Lowe, S.W., and Giaccia, A.J. 1996. Hypoxia-mediated selection of cells with diminished apoptotic potential in solid tumours. Nature 379: 88-91.

Han, J., Sabbatini, P., and White, E. 1996. Induction of apoptosis by human $\mathrm{Nbk} / \mathrm{Bik}$, a $\mathrm{BH} 3$-containing protein that interacts with E1B 19K. Mol. Cell. Biol. 16: 5857-5864.

Han, J., Flemington, C., Houghton, A.B., Gu, Z., Zambetti, G.P., Lutz, R.J., Zhu, L., and Chittenden, T. 2001. Expression of bbc3, a pro-apoptotic BH3-only gene, is regulated by diverse cell death and survival signals. Proc. Natl. Acad. Sci. 98: $11318-11323$.

Hanahan, D. and Weinberg, R.A. 2000. The hallmarks of cancer. Cell 100: $57-70$.

Harris, A.L. 2002. Hypoxia-A key regulatory factor in tumour growth. Nat. Rev. Cancer 2: 38-47.

Jeffers, J.R., Parganas, E., Lee, Y., Yang, C., Wang, J., Brennan, J., MacLean, K.H., Han, J., Chittenden, T., Ihle, J.N., et al. 2003. Puma is an essential mediator of p53-dependent and -independent apoptotic pathways. Cancer Cell 4: 321-328.

Kasof, G.M., Rao, L., and White, E. 1998. Btf: A novel deathpromoting transcriptional repressor that interacts with Bcl-2 related proteins. Mol. Cell. Biol. 19: 4390-4404.

Letai, A., Bassik, M.C., Walensky, L.D., Sorcinelli, M.D., Weiler, S., and Korsmeyer, S.J. 2002. Distinct BH3 domains either sensitize or activate mitochondrial apoptosis, serving as prototype cancer therapeutics. Cancer Cell 2: 183-192.

Levine, A.J. 1997. p53, the cellular gatekeeper for growth and division. Cell 88: 323-331.

Lindsten, T., Ross, A.J., King, A., Zong, W.-X., Rathmell, J.C., Shiels, H.A., Ulrich, E., Waymire, K.G., Mahar, P., Frauwirth, K., et al. 2000. The combined functions of the proapoptotic Bcl-2 family members, Bak and Bax, are essential for the normal development of multiple tissues. Mol. Cell 6: 1389-1399.

Liu, S., Weaver, D.L., and Taatjes, D.J. 1997. Three-dimensional reconstruction by confocal laser scanning microscopy in routine pathologic specimens of benign and malignant lesions of the human breast. Histochem. Cell Biol. 107: 267-278.

Lowe, S.W., Jacks, T., Housman, D.E., and Ruley, E.H. 1994. Abrogation of oncogene-associated apoptosis allows transformation of p53-deficient cells. Proc. Nat1. Acad. Sci. 91: 2026-2030.

Maffini, M.V., Ortega, H.H., Stoker, C., Giardina, R.H., Luque, E.H., and Munoz de Toro, M.M. 2001. Bcl-2 correlates with tumor ploidy and nuclear morphology in early stage prostate carcinoma. A fine needle aspiration biopsy study. Pathol. Res. Pract. 197: 487-492.

Minn, A.J., Boise, L.H., and Thompson, C.B. 1996. Expression of $\mathrm{Bcl}-\mathrm{X}_{\mathrm{L}}$ and loss of $\mathrm{p} 53$ can cooperate to overcome a cell cycle checkpoint induced by mitotic spindle damage. Genes \& Dev. 10: 2621-2631.

Nakano, K. and Vousden, K. 2001. PUMA, a novel proapoptotic gene, is induced by p53. Mol. Cell 7: 683-694.

Perez, D. and White, E. 2003. E1A sensitizes cells to TNF-a by down-regulating c-FLIP . J. Virol. 77: 2651-2662.

Puthalakath, H. and Strasser, A. 2002. Keeping killers on a tight leash: Transcriptional and post-translational control of the pro-apoptotic activity of BH3-only proteins. Cell Death Differ. 9: 505-512.

Rao, L., Debbas, M., Sabbatini, P., Hockenberry, D., Korsmeyer, S., and White, E. 1992. The adenovirus E1A proteins induce apoptosis which is inhibited by the E1B $19 \mathrm{~K}$ and Bcl-2 proteins. Proc. Nat1. Acad. Sci. 89: 7742-7746.

Reed, J.C. 1998. Bcl-2 family proteins. Oncogene 17: 3225-3236.
Reimertz, C., Kogel, D., Rami, A., Chittenden, T., and Prehn, J.H. 2003. Gene expression during ER stress-induced apoptosis in neurons: Induction of the BH3-only protein Bbc3/ PUMA and activation of the mitochondrial apoptosis pathway. J. Cell Biol. 162: 587-597.

Southern, J.F., Warshaw, A.L., and Lewandrowski, K.B. 1996. DNA ploidy analysis of mucinous cystic tumors of the pancreas. Correlation of aneuploidy with malignancy and poor prognosis. Cancer 77: 58-62.

Storchova, Z. and Pellman, D. 2004. From polyploidy to aneuploidy, genome instability and cancer. Nat. Rev. Mol. Cell Biol. 5: 45-54.

Sudbo, J., Lippman, S.M., Lee, J.J., Mao, L., Kildal, W., Sudbo, A., Sagen, S., Bryne, M., El-Naggar, A., Risberg, B., et al. 2004. The influence of resection and aneuploidy on mortality in oral leukoplakia. N. Engl. J. Med. 350: 1405-1413.

Sundararajan, R. and White, E. 2001. E1B 19K blocks Bax oligomerization and tumor necrosis factor alpha-mediated apoptosis. J. Virol. 75: 7506-7516.

Therman, E., Sarto, G.E., and Buchler, D.A. 1983. The structure and origin of giant nuclei in human cancer cells. Cancer Genet. Cytogenet. 9: 9-18.

Trentin, J.J., Yabe, Y., and Taylor, G. 1962. The quest for human cancer viruses. Science 137: 835-841.

Villunger, A., Michalak, E.M., Coultas, L., Mullauer, F., Bock, G., Ausserlechner, M.J., Adams, J.M., and Strasser, A. 2003. p53- and drug-induced apoptotic responses mediated by BH3-only proteins puma and noxa. Science 302: 1036-1038.

Wei, M., Zong, W.-X., Cheng, E., Lindsten, T., Panoutsakopoulou, V., Ross, A., Roth, K., MacGregor, G., Thompson, C., and Korsmeyer, S. 2001. Proapoptotic Bax and Bak: A requisite gateway to mitochondrial dysfunction and death. Science 292: 727-730.

White, E. 1994. p53, guardian of Rb. Nature 371: 21-22.

. 2001. Regulation of the cell cycle and apoptosis by the oncogenes of adenovirus. Oncogene 20: 7836-7846.

Yu, J., Zhang, L., Hwang, P., Kinzler, K., and Vogelstein, B. 2001. PUMA induces the rapid apoptosis of colorectal cancer cells. Mol. Cell 7: 673-682.

Zong, W.-X., Lindsten, T., Ross, A.J., MacGregor, G.R., and Thompson, C.B. 2001. BH3-only proteins that bind pro-survival Bcl-2 family members fail to induce apoptosis in the absence of Bax and Bak. Genes \& Dev. 15: 1481-1486. 


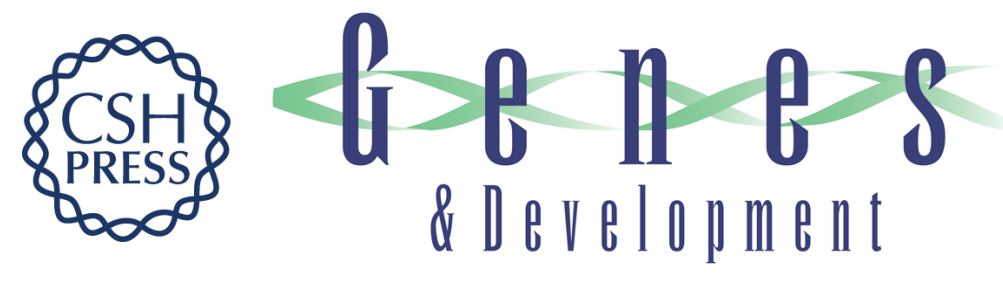

\section{Hypoxia and defective apoptosis drive genomic instability and tumorigenesis}

Deirdre A. Nelson, Ting-Ting Tan, Arnold B. Rabson, et al.

Genes Dev. 2004, 18:

Access the most recent version at doi:10.1101/gad.1204904

$\begin{array}{ll}\text { References } & \begin{array}{l}\text { This article cites } 46 \text { articles, } 18 \text { of which can be accessed free at: } \\ \text { http://genesdev.cshlp.org/content/18/17/2095.full.html\#ref-list-1 }\end{array}\end{array}$

License

Email Alerting

Receive free email alerts when new articles cite this article - sign up in the box at the top Service right corner of the article or click here.

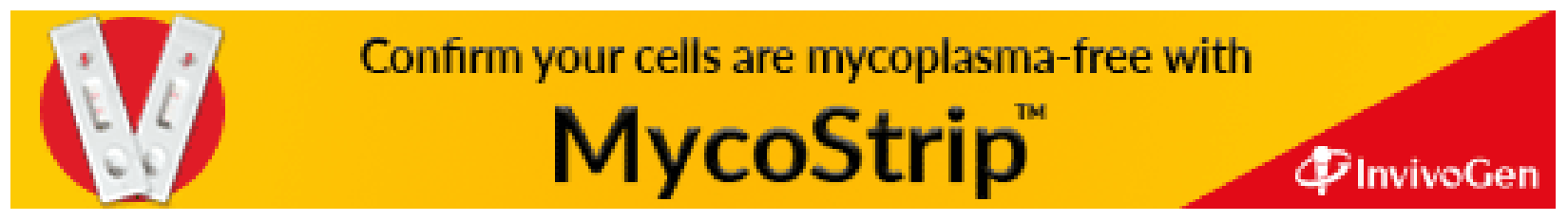

